\title{
Microencapsulation of Probiotics by Calcium Alginate and Gelatin and Evaluation of its Survival in Simulated Human Gastro-Intestinal Condition
}

\author{
Smitha Mathews* \\ Department of Zoology, Assumption College (Autonomous), Chanaganacherry, \\ Kottayam, Kerala, India \\ *Corresponding author
}

\section{A B S T R A C T}

\section{Keywords}

Microencapsulation, Probiotic, Calcium alginate-gelatin, Inulin, Lactulose, Simulated gastrointestinal condition.

Article Info

Accepted:

15 March 2017

Available Online:

10 April 2017
In the recent past, there has been an explosion of probiotic health-based products. However, there are many reports indicated that there is poor survival of probiotic bacteria in these products. Further, the survival of these bacteria in the human gastro-intestinal system is questionable. Providing probiotic living cells with a physical barrier against adverse environmental conditions is therefore an approach currently receiving considerable interest. Microencapsulation being one of the most modern methods has considerable effects on probiotic survival. In this study Lactobacillus acidophilus (NCDC 014) and Lactobacillus casei (NCDC 018)) were encapsulated using calcium alginate-gelatin and prebiotics (inulin and Lactulose) via extrusion technique, and were incubated in simulated gastric juice (along with pepsin, $\mathrm{pH}=1.5$ ) for 2 hours and simulated intestinal juice (along with bile salts, $\mathrm{pH}=8$ ) for 4 hours at $37^{\circ} \mathrm{C}$. . The results indicated that the survival of microencapsulated probiotic increased significantly in simulated gastro-intestinal condition. In general, this study indicated that microencapsulation with alginate-gelatin with prebiotic could successfully and significantly protect $L$. acidophilus and L. casei against adverse condition of simulated human gastro-intestinal condition and offers an effective means of delivery of viable bacterial cells to the colon.

\section{Introduction}

Probiotics are defined as live microorganisms which, when administrated in adequate amounts confer health benefit on humans (FAO, 2001). Bacteria belonging to genera Bifidobacterium and Lactobacillus are often used as probiotic supplements (Homayouni, 2008). With potential health advantages such as alleviation of symptoms of lactose malabsorption, cancer suppression, resistance to infectious gastro-intestinal disease, and improving digestion (Anal et al., 2007; Aragon-Alegro et al., 2007; Shah, 2007). The prebiotics are non-digestible food ingredients that affect the host by selectively stimulating the growth and activity of bacteria in the colon. The most frequently studied examples are inulin and FOS (fructo-oligosaccharides) are known to have a positive effect on human health and promote the survival of probiotic bacteria (Capela et al., 2006).

Microencapsulation with hydrocolloids as one of the most modern methods has remarkable effects on probiotic survival. Encapsulation process is a promising technique for probiotics protection against adverse 
conditions to which probiotics can be exposed (Mokarram et al., 2009). Several studies have been carried out investigating the protective role of this technique. Carbohydrate polymers such as alginate have been used in various microencapsulation procedures (Shu and $\mathrm{Zhu}$ 2002). Alginate is a natural heteropolysaccharide composed of Dmannuronic and L-glucuronic acid residues joined linearly by (1-4) glycosides linkages. Different studies have shown that calcium alginate microcapsules are better protected in the presence of coating polymers and prebiotics such as resistant starch, with the increase in survival of bacteria, under different conditions than when bacteria were tested in the free states (Donthidi et al., 2010).

Gelatin is a protein derived from denatured collagen that contains high levels of hydroxyproline, proline and glycine and is useful as a thermally reversible gelling agent for encapsulation. Gelatin was selected here because of its excellent membrane-forming ability, biocompatibility and non-toxicity. The applicability of gelatine as a hydrogel matrix is limited because of its low network rigidity. However, its physical properties can be improved through the addition of crosslinking agents. Because of its amphoteric nature, it also is an excellent candidate for cooperation with anionic polysaccharides such as alginate and so on (Wenrong and Griffiths, 2000). However, alginate microcapsules (calcium alginate) are chemically susceptible to disintegration in the presence of excess monovalent ions, $\mathrm{Ca}^{2+-}$ chelating agents such as phosphate and citrate and harsh chemical conditions such as those of low pH (Krasaekoopt et al., 2004; Shu and Zhu, 2002; Lee et al., 2004).

Furthermore, this study was undertaken on the alginate and alginate-gelatin encapsulation of probiotic bacteria with inulin and Lactulose as a prebiotic compound. The purpose of this study was to enhance the effectiveness of alginate microencapsulation by gelatin and prebiotics (Inulin and Lactulose) and to evaluate their ability to improve the survival of L. acidophilus and L. casei during exposure to conditions simulated with that of human gastro intestinal environment human gastrointestinal condition.

\section{Materials and Methods}

\section{Experimental set up}

\section{Preparation of cell suspension}

Lyophilized cultures of Probiotic bacteria, Lactobacillus acidophilus (NCDC 014) Lactobacillus casei (NCDC 018) were obtained from National Institute of Dairy, Karnal, Haryana. Lyophilized cells were inoculated in MRS broth (de Man-RogasaSharpe) for $24 \mathrm{~h}$ under aerobic conditions at $37^{\circ} \mathrm{C}$.Biomasses were then harvested by centrifuging at $5000 \mathrm{rpm}$ for $10 \mathrm{~min}$ at $4{ }^{\circ} \mathrm{C}$. The cultures were then washed twice by sterile saline solution $(0.9 \%)$ and used in the microencapsulation process (Mokarram et al., 2009).

\section{Microencapsulation procedure}

All glassware and solutions used in the protocols were sterilized at $121^{\circ} \mathrm{C}$ for $15 \mathrm{~min}$. The the preparation of encapsulated micro capsules was a modified version of methods basically reported by Donthidi et al., in 2010 and Sultana et al., in 2000. Briefly, $2 \mathrm{~g}$ sodium alginate was added to $100 \mathrm{~mL}$ distilled water and boiled until it formed a gel, then $2 \%$ gelatin was also added (Hi media, Mumbai) and required concentrations of inulin $(1 \%)$ and Lactulose $(1 \%)$ were added separately and stirred until they were dissolved or dispersed. Then probiotic cultures of each bacterial species were transferred to the carrier solutions with 
stirring under sterile conditions to ensure uniform distribution of the cells.

\section{Preparation of alginate microspheres}

The conditions used in the experimental work for the probiotic cells encapsulation were: a) $2 \%$ alginate; b) $2 \%$ alginate $+1 \%$ Lactulose; c) $2 \%$ alginate $+1 \%$ inulin; d) $2 \%$ alginate + $2 \%$ gelatine; e) $2 \%$ alginate $+2 \%$ gelatine $+1 \%$ Lactulose; f) $2 \%$ alginate $+2 \%$ gelatine $+1 \%$ inulin. To form capsules, a cell suspension (equivalent of $10^{8} \mathrm{CFU} / \mathrm{g}$ ) was mixed with a $60 \mathrm{ml}$ of $20 \mathrm{~g} / \mathrm{L}$ alginate-gelatin with or without inulin or lactulose and the mixture was dripped into a solution containing $\mathrm{CaCl}_{2}$. The $\mathrm{CaCl}_{2}$ concentration was at $0.1 \mathrm{M}$ and dripping was achieved with a sterile syringe. The distance between syringe and $\mathrm{CaCl}_{2}$ solution was $10 \mathrm{~cm}$. The droplets formed gel spheres instantaneously. Microspheres were hardened $30 \mathrm{~min}$ in $\mathrm{CaCl}_{2}$, and then rinsed with sterile $\mathrm{NaCl}(8.5 \mathrm{~g} / \mathrm{L})$.

\section{Preparation of simulated gastric and} intestinal juices and inoculation of cells

The simulated juices were prepared according to Brinques et al., 2011 and Michida et al., in 2006. Simulated gastric juices were prepared by dissolving pepsin (Himedia, Mumbai) in sterile sodium chloride solution $(0.5 \%, \mathrm{w} / \mathrm{v})$ to a final concentration of $3.0 \mathrm{~g} / \mathrm{L}$ and adjusting the $\mathrm{pH}$ to 1.5 with hydrochloric acid. Simulated intestinal juices were prepared in sterile sodium chloride solution $(0.5 \%$, w/v), with $4.5 \%$ bile salts (Oxoid, Basingstoke, UK) and adjusting the $\mathrm{pH}$ to 8.0 with sterile $\mathrm{NaOH}(0.1 \mathrm{M})$. Both solutions were filtered for sterilization through a 0.22 $\mu \mathrm{m}$ membrane. The probiotic bacteria L.acidophilus and L. casei were inoculated to the simulated gastro-intestinal juice individually in six different forms, nonencapsulated, encapsulated with calcium alginate and calcium alginate-gelatin coated with inulin or Lactulose as prebiotic. Further one gram of freshly encapsulated bacteria samples or $1 \mathrm{~mL}$ of cell suspensions (free cells) were gently mixed with $10 \mathrm{~mL}$ of sterile simulated gastric juice for 2 hours at $37^{\circ} \mathrm{C}$ and followed by inoculation in sterile simulated intestinal juice and incubated at 37 ${ }^{\circ} \mathrm{C}$ for 4 hours.

\section{In vitro release studies (GIT)}

To examine the release behaviour of $L$. acidophilus and L. casei from microcapsules in GIT in vitro, $1 \mathrm{ml}$ of free bacteria and 1 gram encapsulated samples (probiotic bacteria with or without prebiotic and alginate and or gelatin as encapsulating material) were added to $50 \mathrm{ml}$ SGF (pH 1.5) and incubated at physiological temperature $\left(37^{\circ} \mathrm{C}\right)$ for $2 \mathrm{~h}$ and subsequently transferred into $\mathrm{SIF}(\mathrm{pH} 8)$ for another $4 \mathrm{~h}$. At specific time intervals (1 hour), $2.0 \mathrm{ml}$ aliquots were removed and absorbance was measured at $600 \mathrm{~nm}$ in triplicate.

\section{Release of entrapped bacteria}

The capsules containing probiotic bacteria were released by citrate buffer $(\mathrm{pH}=6.0,1 \%)$ reported by Mokarram et al., in 2009. One gram of capsules was transferred to $9 \mathrm{~mL}$ buffer. The solution was stirred on a shaker (Remi Rotary Shaker, RS-12R) for $15 \mathrm{~min}$ vigorously until the bacteria from the capsules were released completely.. The counts (CFU/g) were determined by plating on MRS agar plates and incubating for $48 \mathrm{~h}$ at $37{ }^{\circ} \mathrm{C}$. The free bacteria were treated similarly. All samples were counted in triplicates.

\section{Encapsulation yield}

Encapsulation yield (EY) i.e. the number of bacterial cells that survived the process and encapsulated inside the microcapsules was calculated as follows: 
$\mathrm{EY}=\left(\mathrm{N} / \mathrm{N}_{0}\right) \times 100$

Where $\mathrm{N}_{0}$ is the number of viable bacteria in $\mathrm{CFU} / \mathrm{ml}$ of culture and $\mathrm{N}$ is the number of viable bacteria in $\mathrm{CFU} / \mathrm{g}$ of microcapsules.

\section{Results and Discussion}

In vitro release studies of $L$. acidophilus and L. case $i$ from the microcapsules

Free cells and Microcapsule samples were treated with SGF and then with SIF to check the continuously release characteristics of L.acidophilus and L. casei in GIT and the results are shown in figure 1.

In SGJ ( $\mathrm{pH} 1.5)$, the release amounts of cells were minor from each sample of the microspheres. Once the samples were transferred from SGF to SIF, the larger amounts and faster release rate of $L$. acidophilus (Fig. 1A) and L. casei (Fig. 1B) cells were found, indicated by increase in absorbance.

For L. acidophilus and L. casei faster release of cells occur when they are encapsulated in alginate- gelatin with inulin as prebiotic followed by lactulose. For both probiotics, alginate- gelatin along with prebiotic gave better protection in gastric juice and better release in intestinal fluid. Further free cells were very susceptible to SIF.

\section{Survival of free and microencapsulated probiotics in simulated gastric juice}

Figures II and III show the viability of free and encapsulated probiotic bacteria during incubation in the simulated gastro-intestinal condition, figure II illustrates that the survival of probiotics was lower in gastric juice and decreased further as the incubation period increased. Exposure to simulated gastric juice for 120 minutes resulted in a considerable decrease in the total number of free Lactobacillus acidophilus (Fig. 2A) and L. casei (Fig. 2B) (only $25.6 \%$ and $17.9 \%$ viability respectively). However, the cell number of microencapsulated Lactobacillus acidophilus and L. casei decreased slightly after 120 min. Alginate -gelatin encapsulated L. acidophilus was observed to exhibit the highest viability $(94.11 \%)$ when inulin was incoperated as prebiotic while greatest viability of $84.94 \%$ was observed for alginate encapsulated $L$. casei without any prebiotic. In the case of both $L$. acidophilus and $L$. casei, the survival of cells in both alginate and alginate-gelatin were found to be higher when compared with free cells (Figure 2A,B). Chávarri et al., in 2010 reported that encapsulation in chitosan-coated alginate microspheres significantly improved the survival of Lactobacillus gasseri and Bifidobacterium bifidum in simulated gastric juice along with pepsin. Many scientists have also reported that the survival rate of bifidobacteria in alginate microcapsules was higher than that of free cells (Hansen et al., 2002; Yu et al., 2001; Woo et al., 1999). Many studies have shown coating the alginate matrix could increase the survival of bacteria due to curbing the diffusion of calcium ions outside of capsules (Chávarri et al., 2010; Mokarram, 2009; Krasaekoopt et al., 2004). Mokarram et al., in 2009 showed that $L$. acidophilus and L. rhamnosus exposed to simulated gastric juice without pepsin had higher viability when encapsulated in calcium alginate with double coating sodium alginate.

They indicated that the reduction of pore size and distribution of gastric juice in double layer membrane lead to limitation of interaction between cells with the gastric juice. According to our study, microcapsules both alginate and gelatin coated along with prebiotic (lactulose or inulin) provided the best protection in simulated gastric juice. Furthermore, the increase in viable counts of bacteria could be attributed to the addition of prebiotic. Alginate and prebiotics such as 
inulin or oligosaccharides tend to be synergistic in gelling and as a result may help maintain and improve the degree of protection to bacterial cells (Capela, 2006).

Survival of free and microencapsulated bacteria in simulated intestinal juice
The effect of the simulated intestinal juice on the viability of the microencapsulated and free probiotic bacteria after treatment with simulated gastric juice is presented in Figure III. The number of free probiotics decreased significantly as the incubation time increased.

Figure.1 O.D of free and microencapsulated Lactobacillus acidophilus (A) and Lactobacillus casei (B) in simulated gastro intestinal juice
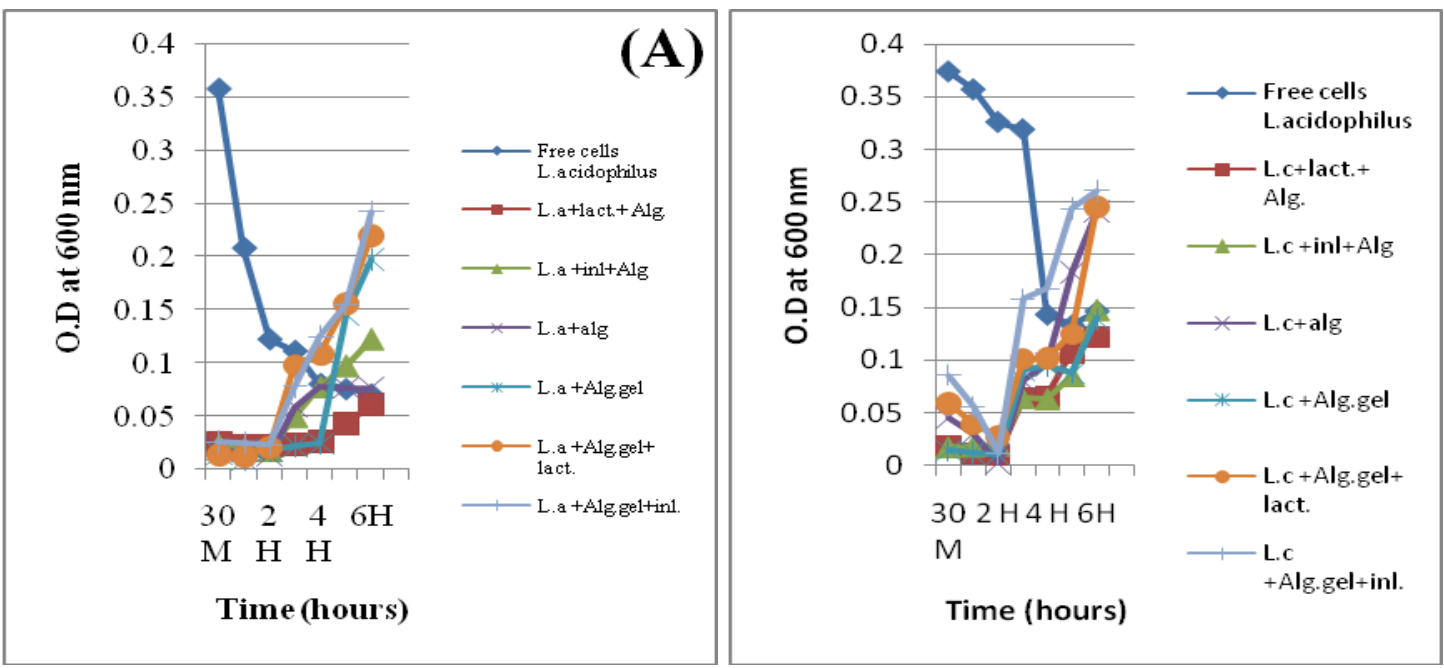

Figure.2 Survival of free and microencapsulated Lactobacillus acidophilus (A) and Lactobacillus casei (B) in simulated gastric juice

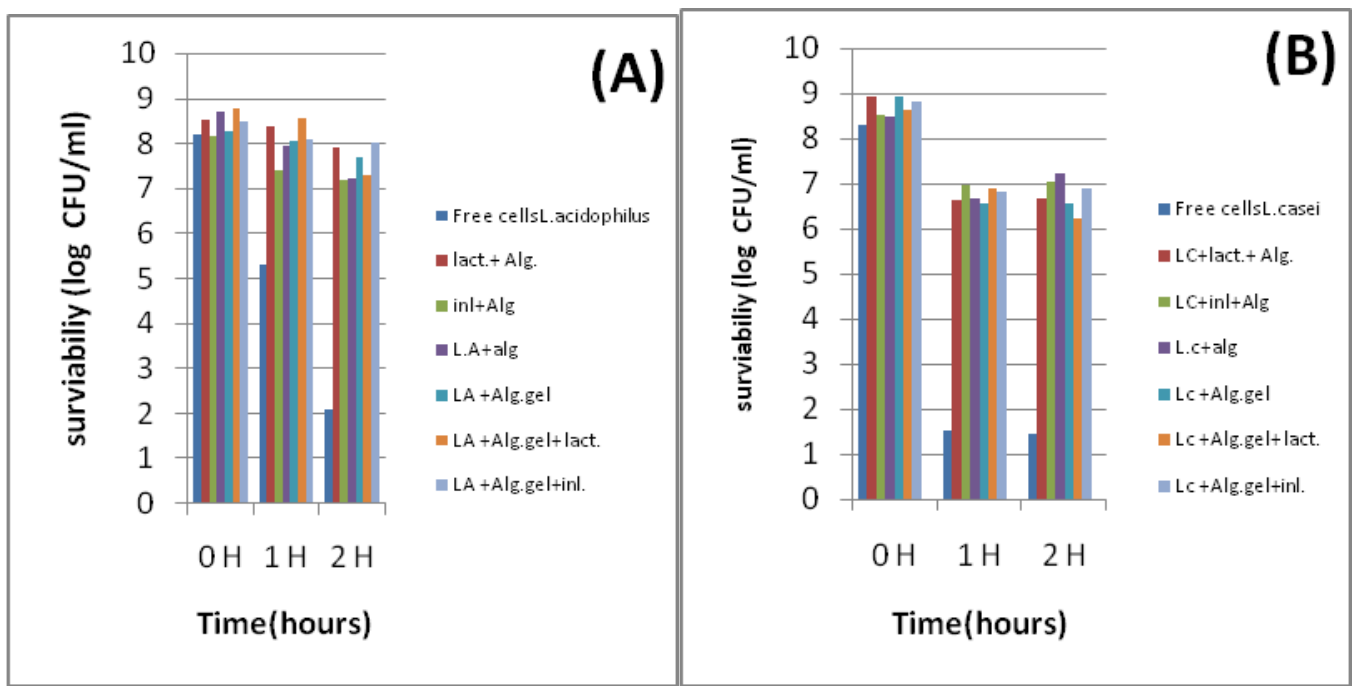


Figure.3 Survival of free and microencapsulated Lactobacillus acidophilus (A) and Lactobacillus casei (B) in simulated intestinal juice
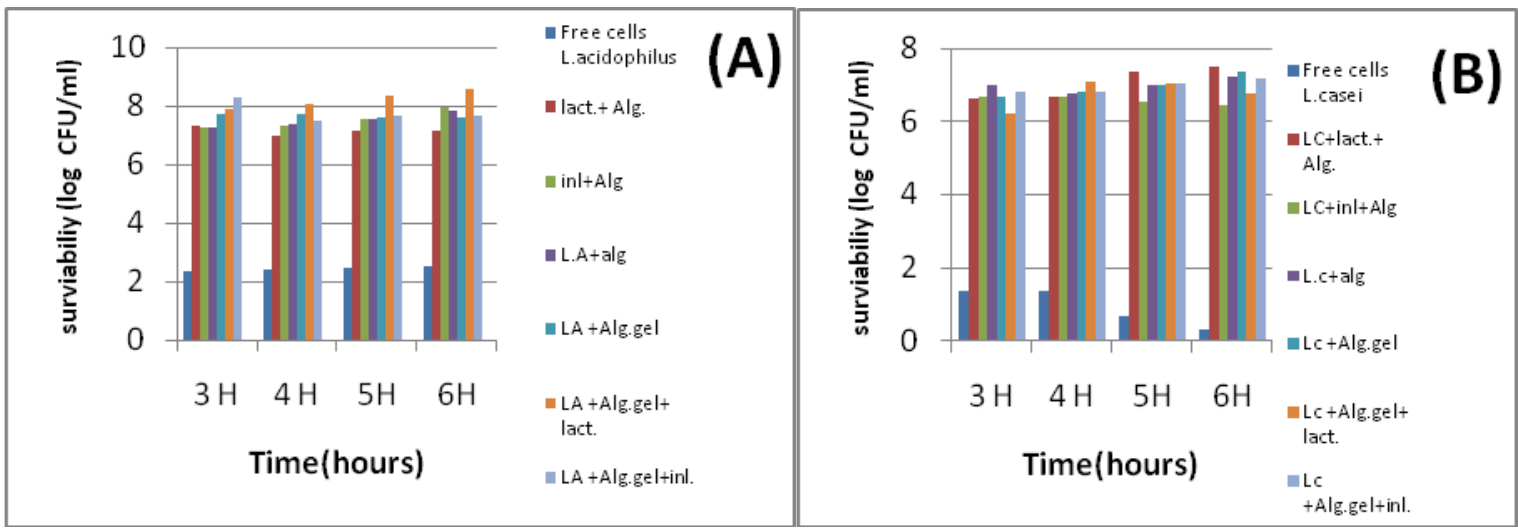

Our result indicated that alginate-gelatin microcapsules with lactulose as prebiotic was most effective in protecting L. acidophilus [Fig. 3 (A)] from simulated intestinal juice with an encapsulation efficiency of $97.38 \%$ followed by inulin encapsulated alginate beads (97.18\%). This underlines the possibility that prebiotics have some effect on encapsulation efficiency. Similarly when L.casei (Fig.3B) after exposure to SGJ for 2 hours when introduced to SIJ, number of free cells decreased drastically with a viability of only $3.6 \%$, while, alginate encapsulated with lactulose showed more survival $(85.9 \%)$.This is in good agreement with the results of Krasaekoopt et al., in 2004 who indicated that the survival of probiotic bacteria was highly enhanced in gastro-intestinal conditions when encapsulated with alginate-chitosan or polyL-lysine. Different studies have shown that calcium alginate microcapsules are better protected in the presence of prebiotics (Sultana et al., 2000, Chen et al., 2005). For L. acidophilus, additional coating of sodium alginate with gelatin provided better survival in simulated gastrointestinal condition, but for L.casei gelatin confers any additional protection than alginate alone. For both probiotic lactulose provided more protection than inulin. All encapsulation method satisfy daily intake of probiotic bacteria above $10^{6}$ living bacteria per milliliter or per gram of the product (Aragon-Alegro et al., 2007).

In conclusion microencapsulation of $L$. acidophilus and L. casei in calcium alginate and calcium alginate-gelatin resulted in better in vitro release and survival of cells in simulated gastro-intestinal condition (along with pepsin and bile salt), compared to free cells. Introduction of prebiotic were found to provide better survival of both probiotic for gastro-intestinal condition. Therefore the applied approach in this study might prove beneficial for the delivery of probiotic cultures to the simulated human gastrointestinal tract. Of the six types of microcapsules with prebiotic in this research, inulin gave better protection in SGJ but lactulose provided the best protection and survival of L. acidophilus and L. casei cells in simulated gastro-intestinal condition. Survival of free L. acidophilus and L. casei drastically decreased due to its low acid and bile resistance. In summary, the microencapsulation method reported in this paper under optimum conditions proved to be very efficient in increasing the viability of probiotic bacteria compared to nonencapsulated free cells. Alginate-gelatin microcapsules might be potentially used as a safe and protective delivery vehicle for administering viable probiotic bacteria. Future 
research researches in this realm may be focused on developing appropriate equipments which can provide potential improvements in the microencapsulation. Related researches are required to assess the efficacy of microencapsulation in the gastrointestinal tract using animal models.

\section{Acknowledgement}

The author would like to express her sincere gratitude and profound appreciation to University Grants Commission (UGC), New Delhi for providing financial support for this research.

\section{References}

Anal, A.K., and Singh, H. 2007. Recent advances in microencapsulation of probiotics for industrial applications and targeted delivery. Trends Food Sci. Technol., 18: 240-251.

Aragon-Alegro, L.C., Alarcon Alegro, J.H., Roberta Cardarelli, H., Chih Chiu, M., and Isay Saad, S.M. 2007. Potentially probiotic and synbiotic chocolate mousse LWT- Food Sci. Technol., 40:669-675.

Brinques, G.B., and Ayub, M.A.Z. 2011. Effect of microencapsulation on survival of Lactobacillus plantarum in simulated gastrointestinal conditions, refrigeration, and yogurt. J. Food Eng., 103: 123-128.

Capela, P., Hay, T.K.C., and Shah, N.P. 2006. Effect of cryoprotectants, prebiotics and microencapsulation on survival of probiotic organisms in yoghurt and freeze-dried yoghurt. Food Res. Int., 39: 203-211.

Chávarri, M., Marañón, I., Ares, R., Ibáñez, F.C., Marzo, F., andVillarán, M.C. 2010. Microencapsulation of a probiotic and prebiotic in alginate-chitosan capsules improves survival in simulated gastro-intestinal conditions. Int. J. Food Microbiol., 142: 185-189. [PubMed]

Chen, K.N., Chen, M.J., Liu, J.R., Lin, C.W. and Chiu, H.Y. 2005. Optimization of incorporated prebiotics as coating materials for probiotic microencapsulation. J. Food Sci., 70: 260-266.

Donthidi, A.R., Tester, R.F., and Aidoo, K.E. 2010. Effect of lecithin and starch on alginate-encapsulated probiotic bacteria. J. Microencapsul., 27: 67-77.

Food and Agriculture Organization (FAO) of the United Nations, World Health Organization Expert Consultation, 2001. Evaluation of health and nutritional properties of powder milk and live lactic acid bacteria. Rome, Italy.

Hansen, L.T., Allan-Wojtas, P.M., Jin, Y.L. and Paulson, A, T. 2002. Survival of Ca-alginate microencapsulated Bifidobacterium spp in milk and simulated gastrointestinal conditions. Food Microbiol., 19: 35-45.

Homayoun, A., Azizi, A., Ehsani, M.R., Yarmand, M.S and Razavi, S.H. 2008. Effect of microencapsulation and resistant starch on the probiotic survival and sensory properties of synbiotic ice cream. Food Chem., 1: 50-55.

Krasaekoopt, W., Bhandari, B., and Deeth, H.C. 2006. Survival of probiotics encapsulated in chitosan-coated alginate beads in yoghurt from UHT- and conventionally treated milk during storage. LWT- Food Sci. Technol., 39: 177-183.

Krasaekoopt, W., Bhandari, B., and Deeth, H. 2004. The influence of coating materials on some properties of alginate beads and survivability of microencapsulated probiotic bacteria. Int. Dairy J., 14: 737-743.

Lee, J.S., Cha, D.S., and Park, H.J. 2004. Survival of Freeze-Dried Lactobacillus 
bulgaricus KFRI 673 in ChitosanCoated Calcium Alginate Microparticles. J. Agr. Food Chem., 52: 7300-7305. [PubMed]

Michida, H., Tamalampudi, S., Pandiella, S.S., Webb, C., Fukuda, H., and Kondo, A. 2006. Effect of cereal extracts and cereal fiber on viability of Lactobacillus plantarum under gastrointestinal tract conditions. Biochem. Eng. J., 28: 7378.

Mokarram, R.R., Mortazavi, S.A., Najafi, M.B.H., and Shahidi, F. 2009. The influence of multi stage alginate coating on survivability of potential probiotic bacteria in simulated gastric and intestinal juice. Food Res. Int., 42: 1040-1045.

Mounsey, J.S. and O'Riordan, E.D. 2008. Influence of pre-gelatinized maize starch on the rheology, microstructure and processing of imitation cheese. $J$. Food Eng., 84: 57-64.

Shah, N.P. 2007. Functional cultures and health benefits. Int. Dairy J., 17: 1262 1277

Shu, X.Z., and Zhu, K.J. 2002. The release behavior of brilliant blue from calcium- alginate gel beads coated by chitosan: the preparation method effect. Eur. J. Pharm. Biopharm., 53: 193-201. [PubMed]

Sultana, K., Godward, G., Reynolds, N., Arumugaswamy, R., Peiris, P., and Kailasapathy, K. 2000. Encapsulation of probiotic bacteria with alginate-starch and evaluation of survival in simulated gastrointestinal conditions and in yoghurt. Int. J. Food Microbiol., 62: 47-55. [PubMed].

Wenrong, S., Griffiths, M.W. 2000. Survival of bifidobacteria in yoghurt and simulated gastric juice following immobilization in gellan-xanthan beads. Int. J. Food Microbiol., 61: 17-25.

Woo, C., Lee, K.and Heo, T. 1999. Improvement of Bifidobacterium longum stability using cell-entrapment technique. J. Microbiol. Biotechnol., 9: 132-139.

Yu, W.K., Yim, T.B., Lee, K.Y.and Heo, T.R. 2001. Effect of skim milk-alginate beads on survival rate of bifidobactersia. Biotechnol. Bioprocess Eng., 6: 133-138.

\section{How to cite this article:}

Smitha Mathews. 2017. Microencapsulation of Probiotics by Calcium Alginate and Gelatin and Evaluation of its Survival in Simulated Human Gastro-Intestinal Condition. Int.J.Curr.Microbiol.App.Sci. 6(4): 2080-2087. doi: https://doi.org/10.20546/ijcmas.2017.604.245 